\title{
Calcified Cerebral Emboli, A "Do Not Miss" Imaging Diagnosis: 22 New Cases and Review of the Literature
}

\author{
B.S. Walker, L.M. Shah, and A.G. Osborn
}

\begin{abstract}
BACKGROUND AND PURPOSE: Calcified cerebral emboli are a rarely reported but devastating cause of stroke and may be the first manifestation of vascular or cardiac disease. Our aim was to evaluate the diagnosis, prevalence, imaging appearance, presumed embolic source, treatment, and outcome of patients with calcified cerebral emboli.

MATERIALS AND METHODS: Our radiology information system was searched for all CT scans by using keywords "calcified," "emboli," and their permutations. The radiology information system was also searched to identify all "stroke" CT reports to calculate the prevalence of calcified cerebral emboli. We also performed a MEDLINE search to identify all published case reports.

RESULTS: Twenty-two cases were identified from our database, and 48 were cases reported from the literature. The middle cerebral artery was the site of $83 \%$ of calcified emboli. Presumed sources were calcific aortic stenosis (36\%), carotid atherosclerotic plaque (30\%), and mitral annular calcification (11\%). Spontaneous embolism occurred in $86 \%$. Surgical treatment was performed in $34 \%$ of patients. Sixty-four percent of the patients with calcified aortic stenosis underwent aortic valve replacement. Among those with identifiable arterial disease, 53\% underwent endarterectomy. Forty-one percent of patients experienced at least 1 recurrent stroke. The prevalence of calcified cerebral emboli identified on stroke CT scans at our institution was $2.7 \%$. Seventy-three percent of cases were correctly identified. Twenty-seven percent were misdiagnosed on initial interpretation, while $9 \%$ were overlooked on preliminary interpretation.
\end{abstract}

CONCLUSIONS: Calcified cerebral emboli are more common than previously assumed, are frequently overlooked or misinterpreted, affect clinical course when diagnosed, and carry substantial risk for recurrent stroke.

C alcified cerebral emboli are a rarely reported but potentially devastating cause of stroke and may be the first manifestation of vascular or cardiac disease. Proper identification can guide treatment toward preventing future embolic events, neurologic impairment, and death. Noncontrast CT of the head is the most common imaging procedure used as the initial assessment of suspected stroke. The first imaging report of calcified cerebral emboli was published in $1981 .^{1}$ Since then, there have been only 48 cases reported in the literature. ${ }^{1-38}$

The purpose of this study was to evaluate the diagnosis, prevalence, imaging appearance, presumed embolic source, treatment,

Received November 7, 2013; accepted after revision January 1, 2014.

From the Department of Radiology, University of Utah School of Medicine, Salt Lake City, Utah.

Paper previously presented at: Annual Meeting of the American Society of Neuroradiology, May 18-23, 2013; San Diego, California.

Please address correspondence to Brett Walker, MD, Department of Radiology, Harvard Medical School, Brigham and Women's Hospital, 75 Francis Street, Boston, MA 02115; e-mail: brett.walker@imail.org

= Indicates article with supplemental on-line tables.

http://dx.doi.org/10.3174/ajnr.A3892 and outcome of patients with calcified cerebral emboli. We report the first comprehensive review of the literature and present the largest imaging series to date, to our knowledge. We demonstrate that these emboli are more common than previously assumed, are frequently overlooked, and carry substantial risk for recurrent stroke.

\section{MATERIALS AND METHODS}

In this institutional review board-approved study, our institutional radiology information system was searched for all noncontrast head CT scans obtained between 2001 and 2013 by using keywords "calcified," "emboli," and their permutations. Cases were identified and then reviewed by 2 board-certified neuroradiologists.

The radiology information system was also searched retrospectively for 12 consecutive months to identify all CT scan reports that contained the word "stroke" within the indication or body of the report. We reviewed all "stroke" NCCT scans and identified all cases with calcified cerebral emboli to calculate the prevalence among all patients obtaining a NCCT scan for stroke in the index period. 

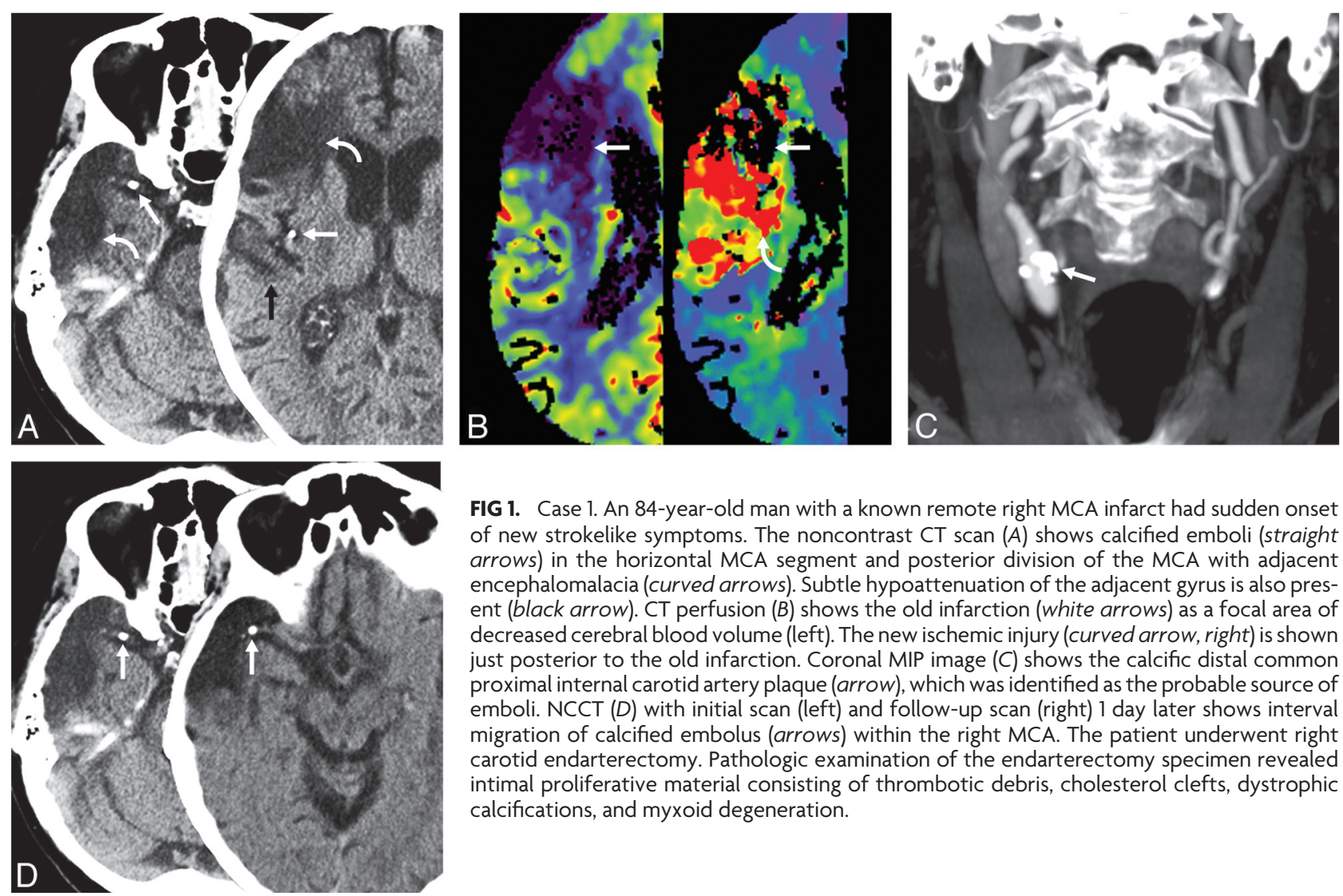

FIG 1. Case 1. An 84-year-old man with a known remote right MCA infarct had sudden onset of new strokelike symptoms. The noncontrast CT scan $(A)$ shows calcified emboli (straight arrows) in the horizontal MCA segment and posterior division of the MCA with adjacent encephalomalacia (curved arrows). Subtle hypoattenuation of the adjacent gyrus is also present (black arrow). CT perfusion (B) shows the old infarction (white arrows) as a focal area of decreased cerebral blood volume (left). The new ischemic injury (curved arrow, right) is shown just posterior to the old infarction. Coronal MIP image (C) shows the calcific distal common proximal internal carotid artery plaque (arrow), which was identified as the probable source of emboli. NCCT (D) with initial scan (left) and follow-up scan (right) 1 day later shows interval migration of calcified embolus (arrows) within the right MCA. The patient underwent right carotid endarterectomy. Pathologic examination of the endarterectomy specimen revealed intimal proliferative material consisting of thrombotic debris, cholesterol clefts, dystrophic calcifications, and myxoid degeneration.
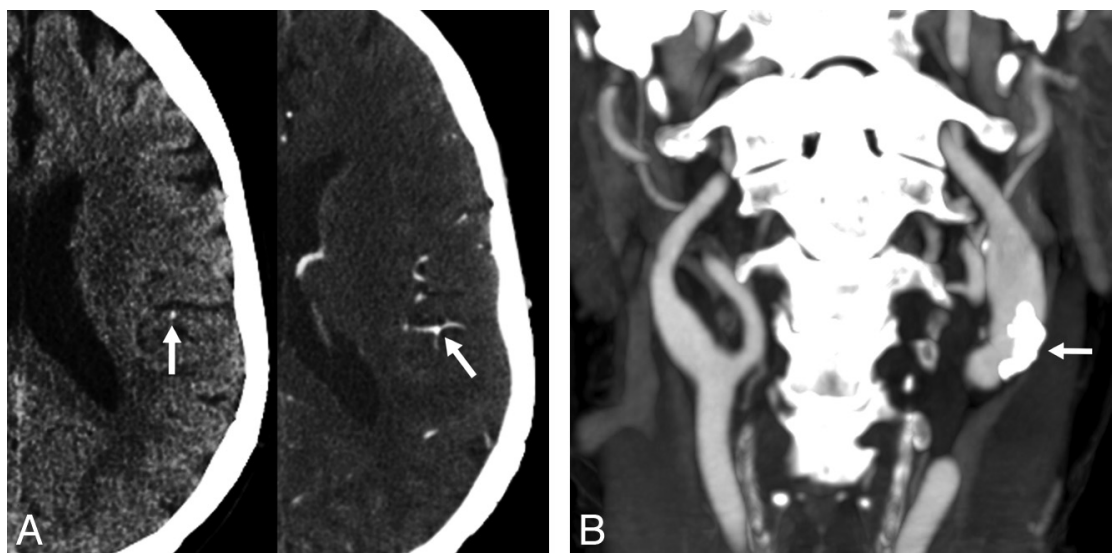

\section{Demographics}

Of the 70 cases, $61 \%$ were men and $39 \%$ women. The mean patient age was 66 years (range, 20-91 years). Sixty-eight $(97 \%)$ of the 70 patients presented with neurologic symptoms concerning for stroke, while 2 patients (3\%) had incidental findings of calcified emboli without acute symptoms. All 70 had noncontrast head CT scans (Fig 1). Twenty-one patients were further evaluated with CT angiography (Fig 2), and 27 patients, by MR imaging (Fig 3).

During the 12-month index period,

FIG 2. Case 2. Axial nonenhanced CT of the head (A, left) in a 59-year-old woman with acuteonset right hemiparesis shows a small hyperattenuated focus (arrow) that measured $101 \mathrm{HU}$, consistent with a calcific embolus within the posterior left frontal lobe. Axial CTA (A, right) confirms the intravascular location of the embolus. Coronal MIP CT angiogram of the neck $(B)$ shows irregular calcified atherosclerotic plaque (arrow) within a dilated left carotid bulb and proximal internal carotid artery. The patient had surgical resection of the left carotid fusiform aneurysm and repair with a graft. Pathologic examination of the specimen disclosed a calcified and thickened wall with approximately $30 \%$ narrowing.

452 stroke head CT scans were obtained in our institution. Twelve of the 22 cases of calcified cerebral emboli occurred during this time. The prevalence of calcified cerebral emboli identified on stroke CT scans at our institution during the index period was $2.7 \%$.

We also performed a MEDLINE search for "calcified" AND "emboli" AND "stroke" to identify all published case reports of calcified cerebral emboli diagnosed on imaging.

\section{RESULTS}

Twenty-two cases were identified from our institutional database, and 48 cases were identified in the literature. Seventy cases were used in our statistical analysis.

\section{Location}

The middle cerebral artery was the most common site with $83 \%$ of all calcified emboli located as follows: M1 (26\%), M2 (24\%), M3 (11\%), and M4 (22\%). The anterior (8\%) and posterior (8\%) cerebral arteries were the next most common sites. One calcified embolus (1\%) lodged within the supraclinoid internal carotid artery.

Multiple calcified emboli were observed in $37 \%$ of patients (Fig 1). Bilateral emboli were observed in $13 \%$ of patients and were 

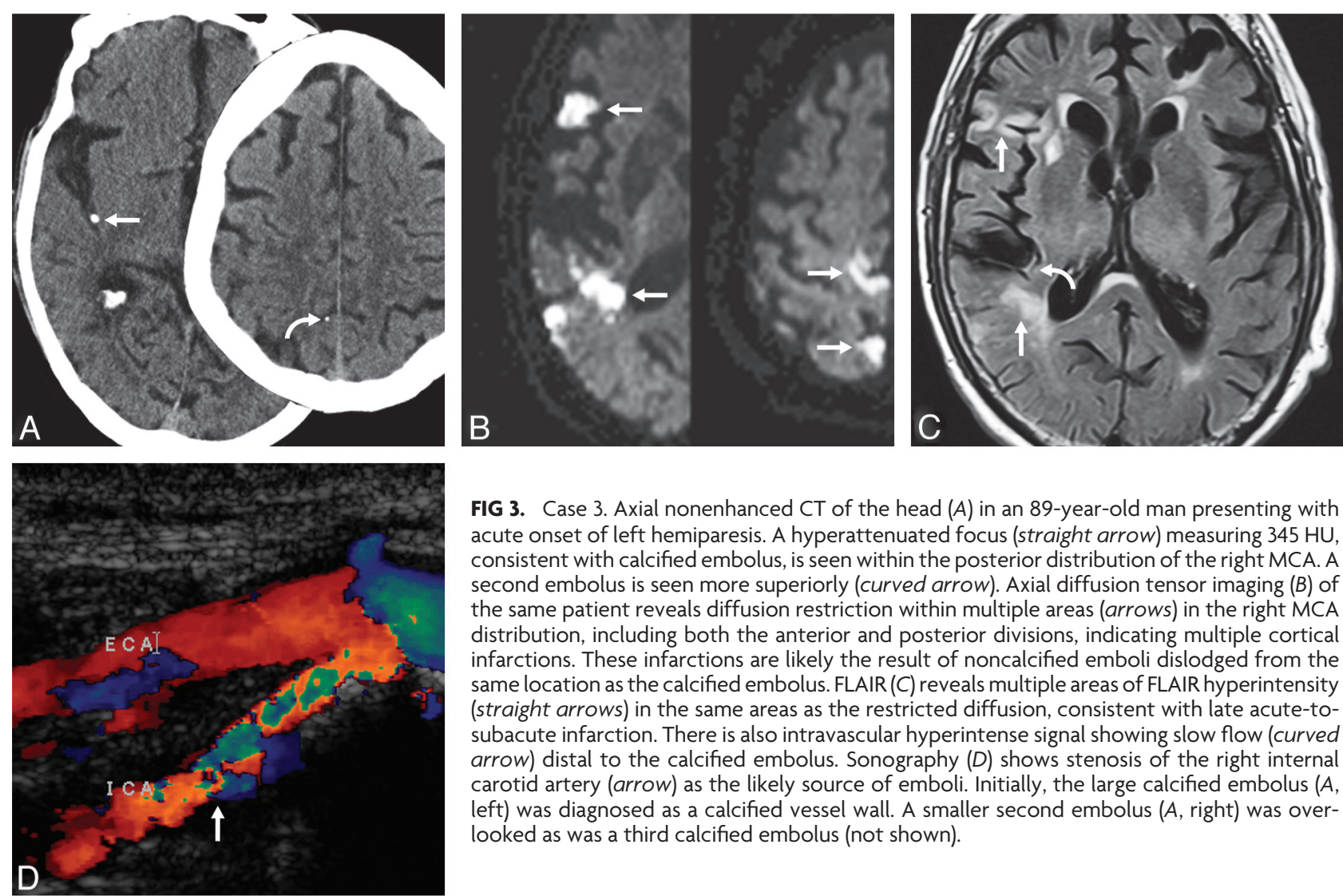

FIG 3. Case 3. Axial nonenhanced CT of the head $(A)$ in an 89-year-old man presenting with acute onset of left hemiparesis. A hyperattenuated focus (straight arrow) measuring $345 \mathrm{HU}$, consistent with calcified embolus, is seen within the posterior distribution of the right MCA. A second embolus is seen more superiorly (curved arrow). Axial diffusion tensor imaging $(B)$ of the same patient reveals diffusion restriction within multiple areas (arrows) in the right MCA distribution, including both the anterior and posterior divisions, indicating multiple cortical infarctions. These infarctions are likely the result of noncalcified emboli dislodged from the same location as the calcified embolus. FLAIR $(C)$ reveals multiple areas of FLAIR hyperintensity (straight arrows) in the same areas as the restricted diffusion, consistent with late acute-tosubacute infarction. There is also intravascular hyperintense signal showing slow flow (curved arrow) distal to the calcified embolus. Sonography $(D)$ shows stenosis of the right internal carotid artery (arrow) as the likely source of emboli. Initially, the large calcified embolus ( $A$, left) was diagnosed as a calcified vessel wall. A smaller second embolus ( $A$, right) was overlooked as was a third calcified embolus (not shown).

equally distributed between hemispheres. The maximum number of calcified cerebral emboli was 6, with an average of 1.7. Repeat imaging, when available, demonstrated distal migration of calcified emboli in $14 \%$ of cases. Following intravenous thrombolytic administration, 4 patients had lysis of a calcified embolus with distal migration of multiple calcified fragments that were identified on repeat scan.

\section{Size, Density, and Configuration}

The average diameter of emboli was $2.5 \mathrm{~mm}$ with a range of 1.0-5.5 $\mathrm{mm}$. Mean embolus attenuation was $162 \mathrm{HU}$, with a range from 79 to $435 \mathrm{HU}$. All emboli were either round (82\%) or ovoid (18\%).

\section{Source}

Potential embolic sources were identified by using echocardiography, sonography, and CT angiography. Presumed sources were calcified aortic stenosis $(36 \%)$, carotid atherosclerotic plaque $(30 \%)$, mitral annular calcification (11\%), aortic arch atherosclerotic plaque $(4 \%)$, brachiocephalic artery atherosclerotic plaque $(3 \%)$, and vertebral atherosclerotic plaque (1\%). An embolic source was not reported in $14 \%$ of patients.

Eighty-six percent of calcified emboli were spontaneous, while $14 \%$ were identified as nonspontaneous events: $9 \%$ following left heart catheterization, $3 \%$ following carotid artery manipulation, and $3 \%$ following cardiopulmonary resuscitation.

\section{Treatment}

Heparin therapy was administered in $24 \%$ of all patients. Thrombolytic therapy was administered to 9 patients (13\%). Postembolic surgical treatment was performed in 24 (34\%) patients. Sixty-four per- cent of surviving patients with calcified aortic stenosis underwent successful aortic valve replacement. Among those with identifiable arterial disease, 53\% underwent successful endarterectomy.

\section{Outcome}

Data from the 22 cases at our institution showed that $43 \%$ of patients with initial CT evidence of calcified cerebral emboli subsequently experienced recurrent stroke, defined as $>1$ hospital presentation for stroke symptoms or imaging evidence of prior infarct on the initial study.

Nine patients received intravenous thrombolytic therapy with symptom improvement in 4 (44\%) of 9 . Residual neurologic impairment from calcified emboli occurred in 33\% of cases. Complete neurologic recovery was seen in $29 \%$. Sixteen percent of patients died during the initial hospitalization. In $23 \%$ of cases, there was no record or follow-up of neurologic outcome.

\section{Diagnosis}

Of the 22 cases at our institution, $73 \%$ were correctly identified as having calcified emboli on NCCT. Twenty-seven percent were misdiagnosed on initial NCCT interpretation as having hemorrhage, infectious, traumatic, or vascular calcification (Fig 3). These were only correctly diagnosed on the review for this study. Nine percent were overlooked on preliminary interpretation by the on-call radiology resident.

\section{DISCUSSION}

Noncontrast CT is the most frequently used technique to screen patients with a clinical diagnosis of acute "stroke." Initial NCCT 
scan findings - even those obtained in the first 6 hours following ictus - are abnormal in most patients with acute ischemic stroke. Positive imaging findings include a hyperattenuating vessel with intraluminal thrombus (eg, the "attenuated MCA" sign and "dot" sign), cortical and/or basal ganglia edema with blurring of graywhite matter interfaces (eg, the "insular ribbon" and "disappearing basal ganglia" signs), and early mass effect with sulcal effacement. ${ }^{39}$ To these widely recognized signs, we add a frequently overlooked but very important finding, calcified cerebral emboli.

Calcified emboli as a specific cause of stroke have been considered rare, with just 48 cases reported in the literature since the first description in $1981 .^{1}$ In a single calendar year, we identified $\geq 1$ calcified emboli in $2.7 \%$ of all patients undergoing NCCT with the clinical indication of stroke. We also found that patients with documented calcified emboli are at significant risk for recurrent embolic infarcts, with nearly half $(43 \%)$ experiencing at least 1 repeat stroke.

While previous reports have suggested that calcified cerebral emboli most commonly occur following manual dislodgement (eg, left heart catheterization, valve surgery, carotid artery manipulation), our data suggest that most of such strokes (86\%) are spontaneous and occur in the absence of such provoking events. ${ }^{4}$

A putative embolic source could be identified in $86 \%$ of all reported cases. Cardiac valvular disease was more common than carotid atheromatous disease, with calcific aortic stenosis 3 times as common as mitral valve disease as the embolic source. More than one-third $(34 \%)$ of patients with calcified emboli subsequently underwent a surgical intervention. Valve replacement was performed in nearly two-thirds of those with calcific aortic stenosis, and endarterectomy was performed in $53 \%$ of those with arterial disease.

Calcified cerebral emboli are often small (2-3 $\mathrm{mm}$ in diameter) and can be easily overlooked or mistaken for other diseases. More than one-quarter of cases at our institution were initially missed entirely or misinterpreted on initial evaluation. Common misdiagnoses included hemorrhage, vessel wall calcification, or infectious residua. All calcified emboli were significantly more attenuated ( $162 \mathrm{HU}$ ) than intraluminal thrombi (typically 50-70 $\mathrm{HU}$ ) and were round or ovoid (not tubular or linear like vascular wall calcification). In no cases were parenchymal calcifications suggestive of neurocysticercosis or other infections identified.

Cardioembolic stroke is considered the most severe ischemic stroke subtype, with a high risk of recurrence and high mortality. ${ }^{40}$ Patients with calcified cerebral emboli identified on NCCT should undergo further imaging to determine an embolic source because timely surgical intervention may prevent repeat ischemic episodes.

The retrospective nature of our radiology information system search may have led to bias because there is an increased sensitivity to identification of calcified emboli in our review of cases for this project. The residents and faculty at our institution have been educated on these findings and, therefore, have an increased awareness of identifying calcified emboli. This is evidenced by the fact that our index year accounts for more than half of all institutional cases in a 13-year period. Because we identified cases on the basis of search terms alone, it is assumed that a large number of cases were not identified that would otherwise have been included had we visually searched through the thousands of NCCT scans obtained for stroke during the 13-year study period. This limitation suggests that reported calcified emboli in our radiology information system are probably a gross underestimation of the real disease prevalence.

\section{CONCLUSIONS}

Calcified cerebral emboli are more common than previously assumed and occur in nearly 3\% of patients obtaining a head CT scan for stroke. They are also frequently misinterpreted or overlooked. Calcified cerebral emboli carry a substantial risk for recurrent stroke with almost half of all patients experiencing a subsequent event. When properly diagnosed, patients with calcified cerebral emboli may be able to undergo surgical correction of underlying cardiac or vascular disease to prevent recurrence. These findings support the need for increased recognition of calcified cerebral emboli and further imaging to establish a presumed source of emboli.

\section{ACKNOWLEDGMENTS}

The authors acknowledge and thank LuAnn Brigham for her valuable expertise in performing database queries.

Disclosures: Anne G. Osborn—UNRELATED: Board Membership: Amirsys, Amirsys Publishing, Employment: Amirsys Publishing (CEO), Payment for Lectures (including service on Speakers Bureaus): Mallinckrodt, Comments: Neuroradiology from the Master, Royalties: Amirsys, Comments: book royalties, Stock/Stock Options: Amirsys, Amirsys Publishing, Comments: shareholder.

\section{REFERENCES}

1. Yock DH Jr. CT demonstration of cerebral emboli. J Comput Assist Tomogr 1981;5:190-96

2. Kapila A, Hart R. Calcific cerebral emboli and aortic stenosis: detection of computed tomography. Stroke 1986;17:619-21

3. Rancurel G, Marelle L, Vincent D, et al. Spontaneous calcific cerebral embolus from a calcific aortic stenosis in a middle cerebral artery infarct. Stroke 1989;20:691-93

4. Vernhet H, Torres GF, Laharotte JC, et al. Spontaneous calcific cerebral emboli from calcified aortic valve stenosis. J Neuroradiol 1993;20:19-23

5. O'Donoghue ME, Dangond F, Burger AJ, et al. Spontaneous calcific embolization to the supraclinoid internal carotid artery from a regurgitant bicuspid aortic valve. Neurology 1993;43:2715-17

6. Kirk GR, Johnson JK. Computed tomography detection of a cerebral calcific embolus following coronary catheterization. J Neuroimaging 1994;4:241-42

7. Doiron A, Blais C, Bonneau D. Spontaneous cerebral embolus from a calcified aortic valve. AJR Am J Roentgenol 1996;167:955-56

8. Shanmugam V, Chhablani R, Gorelick PB. Spontaneous calcific cerebral embolus. Neurology 1997;48:538-39

9. Mouton P, Biousse V, Crassard I, et al. Ischemic stroke due to calcific emboli from mitral valve annulus calcification. Stroke 1997;28:2325-26

10. Katsamakis G, Lukovits TG, Gorelick PB. Calcific cerebral embolism in systemic calciphylaxis. Neurology 1998;51:295-97

11. Mohammadkhani M, Schaefer P, Koroshetz W, et al. Mitral annulus calcareous brain emboli. Neurology 2000;54:817

12. Khaw N, Gailloud P. CT of calcific cerebral emboli after carotid manipulation. AJR Am J Roentgenol 2000;174:1467

13. Oliveira-Filho J, Massaro AR, Yamamoto F, et al. Stroke as the first manifestation of calcific aortic stenosis. Cerebrovasc Dis 2000; 10:413-16

14. Kissela BM, Kothari RU, Tomsick TA, et al. Embolization of calcific 
thrombi after tissue plasminogen activator treatment. J Stroke Cerebrovasc Dis 2001;10:135-38

15. Martínez-Fernández E, Gil-Néciga E, Mir P, et al. Spontaneous cerebral calcium embolism [in Spanish]. Rev Neurol 2002;34:354-57

16. Lahey $\mathrm{T}$, Horton S. Massive left atrial calcification and devastating systemic emboli in a patient with chronic renal failure. Am J Kidney Dis 2002;40:416-19

17. Debruxelles S, Sibon I, Rouanet F, et al. Cerebral infraction by calcified embolism: a spontaneous complication of calcified aortic stenosis [in French]. Rev Neurol (Paris) 2004;160(5 pt 1):582-84

18. Halloran JI, Bekavac I. Unsuccessful tissue plasminogen activator treatment of acute stroke caused by a calcific embolus. J Neuroimaging 2004; 14:385-87

19. Gearry RB, Sharr JP, Avery SF. Spontaneous calcific cerebral embolus. Australas Radiol 2005;49:154-56

20. Moustafa RR, Antoun NM, Coulden RA, et al. Stroke attributable to a calcific embolus from the brachiocephalic trunk. Stroke 2006;37:e6-8

21. Kavanagh EC, Fenton DM, Heran MK, et al. Calcified cerebral emboli. AJNR Am J Neuroradiol 2006;27:1996-99

22. Sylaja PN, Hill MD. Stroke due to calcific embolus following coronary angiography. Neurology 2006;67:E16

23. Aoyama T, Ohtaki M, Nomura T, et al. Artery-to-artery embolism due to ruptured calcified carotid plaque [in Japanese]. No Shinkei Geka 2007;35:283-88

24. Bugnicourt JM, Bonnaire B, Lepage L, et al. Stroke due to spontaneous calcified cerebral embolus as presenting feature of calcified aortic stenosis [in French]. J Mal Vasc 2008;33:106-09

25. Schirmer CM, Thaler DE, Malek AM. Stent-mediated wedging of a calcific embolus to recanalize an occluded middle cerebral artery: technical case report. Neurosurgery 2008;63:E180-81, discussion E181

26. Mori M, Wakugawa Y, Yasaka M, et al. Case with multiple brain infarcts due to calcified emboli from a calcified atheroma of the innominate artery [in Japanese]. Brain Nerv 2008;60:955-61

27. Tardy J, Da Silva N, Glock Y, et al. Neurological pictures. Stroke with calcium emboli related to a calcified stenosis of internal carotid artery. J Neurol Neurosurg Psychiatry 2008;79:1273-74
28. Christian BA, Kirzeder DJ, Boyd J, et al. Showered calcific emboli to the brain, the 'salted pretzel' sign, originating from the ipsilateral internal carotid artery causing acute cerebral infarction. Stroke 2009;40:e319-21

29. Okazaki S, Sakaguchi M, Sugiyama Y, et al. Ineffective thrombolytic therapy for calcified cerebral emboli originated from calcified internal carotid artery stenosis [in Japanese]. Rinsho Shinkeigaku 2009;49:281-84

30. Roifman I, Glikstein RS, Rudski L. Spontaneous cerebral embolism in a forty year old man: case report and review of the literature. $J \mathrm{Am}$ Soc Echocardiogr 2009;22:1197.e1-3

31. Acha O, Novo F, Espina B, et al. Spontaneous cerebral calcific embolus from the aortic arch. Clin Neurol Neurosurg 2009;111:862-63

32. Yong SW, Lim TS, Hong JM, et al. Migrating calcification on CT angiography: another sign of an embolic stroke. Eur Neurol 2010;63:126

33. Konishi-Yakushiji M, Yakushiji Y, Kotooka N, et al. Sonographic confirmation of the association between calcified cerebral emboli and mitral annular calcification. J Ultrasound Med 2010;29:1507-10

34. Herskovitz M, Telman G, Carasso S, et al. Ischemic stroke due to a calcified embolus from the mitral annular valve. Neurology 2012;78:931

35. Gschwind M, Binaghi S, Zekeridou A, et al. Neurological picture. Dispersion and 'salted pretzel sign' from thrombolysis of a spontaneous calcified embolus in an acute stroke. J Neurol Neurosurg Psychiatry 2013;84:111-12

36. Chandran V, Pai A, Rao S. Calcified embolism: a rare cause of cerebral infarction. BMJ Case Rep 2013;2013:pii: bcr2013009509

37. Gokhale S, Lahoti S, Rojas R, et al. Tissue plasminogen activator and calcific emboli. JAMA Neurol 2013;70:642-43

38. Dandamudi VS, Thaler DE, Malek AM. Cerebral embolus following chiropractic manipulation in a patient with a calcified carotid artery. J Neuroimaging 2013;23:429-30

39. Osborn AG. Acute cerebral ischemia-infarction. Osborn's Brain: Imaging, Pathology, and Anatomy. Salt Lake City, Utah: Amirsys; 2013:180-91

40. Arboix A, Alió J. Cardioembolic stroke: clinical features, specific cardiac disorders and prognosis. Curr Cardiol Rev 2010;6:150-61 